\title{
DIFFERENCES BETWEEN GALAXIES
}

\author{
SIDNEY VAN DEN BERGH
}

David Dunlap Observatory, University of Toronto, Ontario, Canada, and Institute of Astronomy, University of Cambridge

\begin{abstract}
It is pointed out that the metallicity of galactic and extragalactic globular clusters correlates with the density of the regions in which they were formed. Within individual galaxies the regions of highest density have the highest metallicity.

Some of the differences in family traits between the globulars in the Andromeda Nebula, the Galaxy and the Magellanic Clouds might be understood if the M31 cluster system is $\sim 1 \times 10^{9} \mathrm{yr}$ older and the SMC cluster system $\sim 1 \times 10^{9}$ yr younger than galactic globular clusters.
\end{abstract}

\section{Evidence for Age Differences Between Galaxies}

It is usually assumed that the differences between galaxies along the Hubble sequence are due primarily to differences in initial conditions which led to variations in their rate of evolution, rather than to some being younger than others. There is, however, some evidence which suggests that age differences might also be important. Perhaps the strongest such evidence comes from the study of globular clusters associated with members of the Local Group. Intercomparison of the morphology of the colour-magnitude diagrams of globular clusters in the Galaxy and in the Magellanic Clouds shows a number of significant differences. The most striking of these is that Small Cloud clusters of intermediate metal abundance $(\Delta V \sim 2.5)$ have a much stronger population on the red side of the RR Lyrae variable gap than do clusters of similar metal abundance in the Galaxy (see Table I). According to computations by Rood (1973) such a difference could be due to either of the following two causes: (1) the hydrogen abundance in the Small Cloud might be a few percent greater (i.e. the helium abundance lower) than it is in galactic globular clusters, or (2) the globular clusters in the Small Magellanic Cloud might be $\sim 1 \times 10^{9}$ yr younger than are their galactic counterparts (see Figure 1). Presently available observational data (cf. Peimbert p. 141) do not favour the view that the helium abundance in emission nebulae in the Magellanic Clouds differs from that in the Galaxy, although the small change that is required to account for the differences between the globulars in the Clouds and in the Galaxy probably cannot yet be ruled out with certainty.

Spectral scans of a few strong-lined globulars in M31 by Spinrad and Schweizer (1972) appear to show that these clusters exhibit quite strong hydrogen lines. This suggests that these globulars have strongly developed blue horizontal branches despite the fact that their metallicity is high. According to Rood's computations such an effect could be understood if the globulars in M31 are $\sim 1 \times 10^{9} \mathrm{yr}$ older than are their galactic counterparts. This might possibly indicate that high mass (and high density) galaxies such as M31 collapsed somewhat earlier than did galaxies of lower density and lower mass.

Observations of galactic globular clusters show that many of the metal-poor glob- 
TABLE I

CM diagrams of cloud clusters

\begin{tabular}{|c|c|c|c|c|c|c|}
\hline & $M_{V}$ (H.B.) & $M_{V}(\text { R.G. })^{\mathrm{b}}$ & $\Delta V^{\mathrm{c}}$ & $\mathbf{H B}^{d}$ & $(B-V)_{0 \max }{ }^{\mathrm{e}}$ & Reference \\
\hline \multicolumn{7}{|l|}{$S M C$} \\
\hline NGC $121^{a}$ & 19.5 & 16.8 & 2.7 & $\mathbf{R}$ & 1.6 & Tifft (1963) \\
\hline NGC 339 & $?$ & 16.0: & $?$ & $?$ & 2.2: & Gascoigne (1966) \\
\hline NGC 361 & $?$ & 16.5 & $?$ & $?$ & 2.2 & Arp (1958) \\
\hline NGC 419 & $19.3:^{p}$ & 16.8 & 2.5: & $\mathbf{R}$ & 4.1 & Walker (1972b) \\
\hline L1 & $?$ & 17.0 & $?$ & $?$ & 2.2 & Gascoigne (1966) \\
\hline K 3 & 19.1 & 16.6 & 2.5 & $\mathbf{R}$ & 2.3 & Walker (1970) \\
\hline \multicolumn{7}{|l|}{$L M C$} \\
\hline NGC $1466^{a}$ & 19.1 & 16.3 & 2.6 & B & 1.5 & $\begin{array}{l}\text { Gascoigne (1966) } \\
\text { Hodge (1960a) }\end{array}$ \\
\hline NGC 1783 & 19.2 & 16.8 & 2.4 & B & 1.8 & Gascoigne (1962) \\
\hline NGC 1841 & 19.6 & 16.6 & 3.0 & B & 1.7 & Gascoigne (1966) \\
\hline NGC 1846 & $?$ & 16.0 & $?$ & $?$ & 2.5 & Hodge (1960a) \\
\hline NGC $1978^{a}$ & $?$ & 16.4 & $?$ & $?$ & 2.0 & Hodge (1960b) \\
\hline NGC 2231 & $?$ & 16.5: & $?$ & $?$ & 1.6: & Gascoigne (1966) \\
\hline NGC $2257^{a}$ & 19.2 & 16.1 & 3.1 & B & 1.8 & Gascoigne (1966) \\
\hline
\end{tabular}

a Cluster contains RR Lyrae variables.

b Magnitude of giant branch at $(B-V)_{0}=1.4$

c Difference between $M_{V}$ (H.B.) and $M_{V}$ (R.G.)

d $R$ - most of stars on red side of RR Lyrae gap, $B$ - most stars on blue side of gap.

e Colour of reddest cluster giant star. In some clusters the reddest star might be a field object.

${ }^{\mathrm{P}}$ The colons indicate uncertain values.

ulars at distances $\gtrsim 50 \mathrm{kpc}$ from the galactic nucleus have red horizontal branches. For $R=50 \mathrm{kpc}$ the free-fall time-scale* is $\sim 1 \times 10^{9} \mathrm{yr}$. According to Rood's calculations this is sufficient to account for the observed differences between the horizontal branch morphology of distant globular clusters and that of globulars nearer to the galactic centre.

An evolutionary picture in which the metal-poor globular clusters in the outer halo of the Galaxy are younger than are the clusters nearer to the galactic nucleus is consistent with the model of galactic evolution that has been proposed by Larson (1969).

Two dwarf spheroidal galaxies are known to have red horizontal branches of the NGC 7006 type. According to the calculations by Rood (1973) this indicates that these dwarf spheroidals are slightly younger than the oldest galactic Population II objects. A similar suggestion has previously been made by Castellani et al. (1973) who based their conclusion on the properties of the RR Lyrae variables in dwarf spheroidal systems.

* It should be emphasized that the free-fall time-scale represents a lower limit to the actual collapse time-scale. This is so because the collapse of the Galaxy might have been delayed by turbulent pressure or by radiation pressure from a quasar-like galactic nucleus. 

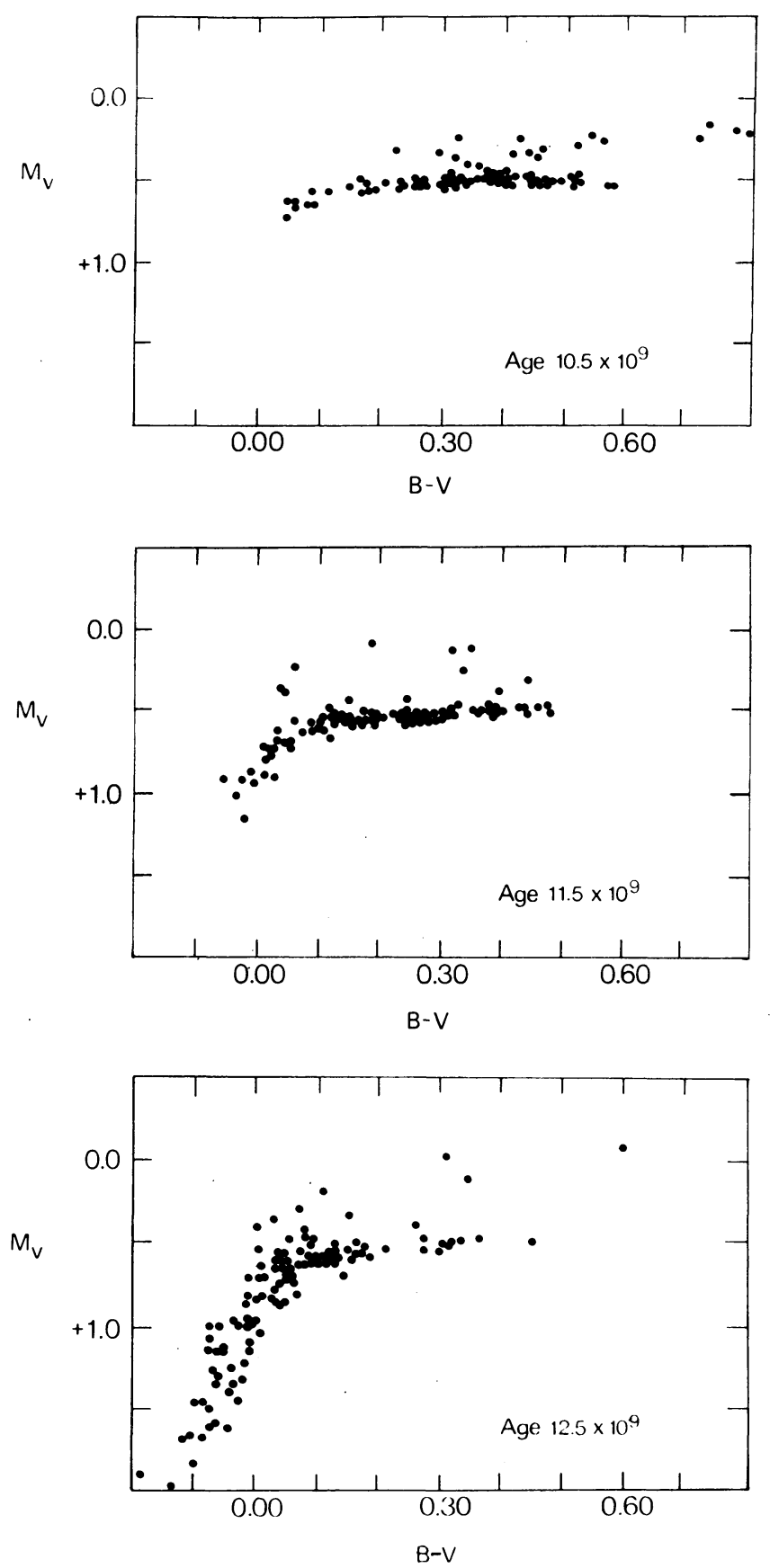

Fig. 1. Dependence of the morphology of the globular cluster horizontal branch on age (in years) for a cluster with $X=0.75$ and $Z=0.001$ according to Rood (1973). Note that the luminosity level of the horizontal branch is insensitive to age. 


\section{Differences Between the Globular Cluster Systems Associated with Different Galaxies}

The globular cluster systems associated with different galaxies exhibit a number of striking differences. Perhaps the most fundamental of these is that the mean metallicity of globular cluster families differs from galaxy to galaxy. The data in Table II seem to show a correlation between the mean metallicity of a cluster family and the mass (or mean density) of the galaxy with which it is associated. All of the globulars associated with the Fornax dwarf spheroidal system appear to be metal-poor. The certain globular clusters (i.e. those that contain RR Lyrae stars) in the Magellanic Clouds, such as NGC 121, 1466, 1978 and 2257, all appear to be moderately metal-poor. The globular clusters associated with the Galaxy exhibit the entire range from extreme metal deficiency to metal abundances close to that of the Sun. The globular clusters near the Andromeda Nebula exhibit the same range in line strength as do those associated with the Galaxy. The mean metallicity of the M31 globulars is, however, considerably greater than is that of the clusters associated with the Galaxy. Finally, recent observations by Ables et al. (1973) appear to show that the globular clusters associated with the giant elliptical galaxy M87 are even redder (and hence presumably metal richer) than are those in M31.

Within the Galaxy, metal-poor globulars appear to have formed predominantly in low density halo regions, whereas metal-rich globulars are mainly found in the high density inner disk and central bulge of the Galaxy. Taken at face value these observations suggest that the metal abundance of globular clusters was mainly determined by the density of the regions in which they were formed.

Intercomparison of the globular clusters in the Galaxy, in the Magellanic Clouds and in M31 provides strong evidence for the conclusion that clusters in different galaxies exhibit differing 'family traits'. For example the well-known correlation between the morphology of the horizontal branch and metallicity of galactic globular

\section{TABLE II}

Relation between mass, line-strength and colour of globular clusters

$\begin{array}{llll}\text { Object } & \mathfrak{M} / \mathfrak{M}_{\odot} & \langle L\rangle & \langle B-V\rangle_{0} \\ & & & \\ \text { Fornax } & & & \\ \text { Galaxy } & 1.3 \times 10^{7} & 0 & 0.67^{\mathrm{b}} \\ \text { Andromeda } & 1.3 \times 10^{11} & 4 & - \\ \text { M87 } & 3.1 \times 10^{11} & 8 & 0.73^{\mathrm{c}} \\ & \sim 1 \times 10^{13} & - & 0.8^{\mathrm{d}}\end{array}$

a $\left(\mathfrak{M} / \mathfrak{M}_{\odot}\right) /\left(L_{V} / L\right)=1$ assumed.

b No galactic reddening correction applied.

c Values refer only to halo clusters (van den Bergh, 1969).

d Colours from Ables et al. (1973). 
clusters does not seem to be valid for M31 or for the Magellanic Clouds. Furthermore, many of the globular clusters in the Magellanic Clouds appear to contain exceedingly red stars. Presumably these are mild carbon stars situated at the tip of the asymptotic giant branch. Probably these stars have managed to mix the carbon formed in their interiors to their surfaces. No stars of this type have been discovered in any galactic globular clusters. Stars with $B-V>2.0$ have also been observed in the Sculptor dwarf spheroidal system (Hodge, 1965). Additional support for the notion that the Population II component in the Magellanic Clouds may exhibit some similarity to the Population II observed in dwarf spheroidals is provided by two types of observations of $\mathrm{W}$ Virginis stars: (1) no long-period ( $P>10$ days) W Virginis stars have so far been discovered in any dwarf spheroidal system. In this respect the dwarf spheroidals resemble the Magellanic Clouds in which W Virginis stars appear to be exceedingly rare. (2) Van Agt (1967) has shown that the short-period W Virginis stars (BL Herculis stars) in dwarf spheroidal systems obey a different period-luminosity relation than do those that occur in galactic globular clusters. Photoelectrically-calibrated photographic observations of a 1.43 day SMC field variable near NGC 121 (Tifft 1963) show that this object must lie on the dwarf spheroidal period-luminosity relation for $\mathrm{BL}$ Herculis stars rather than on that for galactic globular clusters.

\section{Relation Between Metallicity and Mean Density in Galaxies}

Within individual galaxies there is clear-cut evidence for metal abundance gradients. The highest metallicity occurs in the dense cores of galaxies (McClure, 1969). Progressively lower heavy-element abundances are observed as regions of lower density are approached. The correlation between mean metallicity and absolute magnitude (and hence presumably mean density) which is observed in elliptical galaxies (Faber, 1973) also seems to hold for spirals and irregulars. A number of lines of evidence (see Section 4) appear to indicate that young stars in the Magellanic Clouds are mildly metal-deficient compared to those which are currently being formed in the solar vicinity of the Galaxy. Among systems containing a young population component, the correlation between density (or absolute magnitude) and metallicity manifests itself in its most extreme form in the blue dwarf galaxies that have recently been studied by Searle and Sargent (1972).

The relation between metallicity and absolute luminosity is, within the accuracy of the data, the same for elliptical galaxies located inside and outside of clusters (McClure and van den Bergh, 1968). This suggests that either (1) ellipticals in the field have been ejected from clusters or (2) the evolutionary history of a galaxy is determined exclusively by its initial conditions and not by its environment.

It would be very important to gain some theoretical understanding of the reason for the correlation between the metallicity of a stellar system and its density. Such a correlation might be understood if the luminosity function of star formation (Salpeter function) in dense regions favoured high-mass stars that evolved to produce heavy elements. 


\section{Differences among Young Stellar Populations}

Differences in metallicity (but not in helium abundance) are strongly suggested by a number of recent studies of young stellar populations in galaxies. Probably the most direct evidence for differences in chemical composition between galaxies comes from observations of $\mathbf{H}$ il regions. Such observations show that the emission regions in the dwarf galaxy NGC 6822 (Peimbert and Spinrad, 1970) are deficient in nitrogen and oxygen by factors of 6 and 1.7 respectively compared to $H$ II regions near the Sun. In its most extreme form this phenomenon manifests itself in the very low luminosity galaxies studied by Searle and Sargent (1972) in which some heavy elements are $\sim 10$ times less abundant than they are in the Orion Nebula.

Direct observations of the metal abundances in the most luminous stars in the Magellanic Clouds have so far produced rather confusing results. Perhaps the best that can be said is that currently available observational data are not inconsistent with the view that young stars in the Magellanic Clouds are mildly metal-deficient compared to their galactic counterparts. In a series of papers on open clusters in the Magellanic Clouds, published in the late 1950's, Arp was able to show that young and intermediate age clusters in the Clouds exhibit a number of striking differences from their galactic counterparts. Presumably such differences imply that the evolutionary tracks of highly evolved young stars are quite sensitive to chemical composition.

Additional evidence for composition differences between young stellar populations in galaxies of different type may be obtained from observations of the magnitudes of the brightest red and blue stars of Population I. Table III lists the magnitudes of the brightest red and blue stars in all late-type galaxies for which photoelectricallycalibrated magnitudes and accurate distances are available. The data in the Table are

TABLE III

Magnitudes of brightest stars in galaxies

\begin{tabular}{|c|c|c|c|c|c|}
\hline Name & $M_{V}$ & $\begin{array}{l}\text { Brightest } \\
\text { blue star }\end{array}$ & $\begin{array}{l}\text { Brightest } \\
\text { red star }\end{array}$ & $\Delta V$ & Reference \\
\hline Galaxy ${ }^{a}$ & $-20.4:^{b}$ & 5.68 & 7.69 & 2.01 & $\begin{array}{l}\text { Johnson and } \\
\text { Morgan (1955) }\end{array}$ \\
\hline NGC 2403 & -19.3 & $\therefore 18.25^{\mathrm{c}}$ & $19.98^{d}$ & 1.73 & $\begin{array}{l}\text { Tammann and } \\
\text { Sandage (1968) }\end{array}$ \\
\hline LMC & -18.5 & 9.11 & 10.5: e & 1.39 & van den Bergh (1968) \\
\hline SMC & -16.8 & 10.13 & 10.8: e & 0.67 & van den Bergh (1968) \\
\hline NGC 6822 & -15.7 & 16.61 & 16.65 & 0.04 & Kayser (1967) \\
\hline IC 1613 & -14.8 & 17.15 & $16.5: \mathrm{de}$ & -0.65 & Sandage (1971) \\
\hline
\end{tabular}

a Data refer to $\mathrm{h}$ and $\chi$ Persei only.

b $M_{V}$ is derived from $M_{B}=-19.7 \pm 0.3$ (van den Bergh, 1972) and $B-V=0.7$ from $V(\text { rot })_{\max }$ $\simeq 250 \mathrm{~km} \mathrm{~s}^{-1}$ (van den Bergh, 1971).

c $B-V=0.0$ assumed.

d Refers to maximum light.

e $B-V=2.0$ assumed. 
plotted in Figure 2. This figure shows that there is a tight correlation between $\Delta V$, the difference in visual magnitude of the brightest blue and red stars, and the absolute magnitude of the galaxy in which these stars occur. Inspection of the data in Table III shows that the absolute magnitudes of the brightest blue stars in galaxies are very sensitive to the absolute magnitude (and hence presumably to the total Population I content) of the galaxy in which they occur. As Tammann and Sandage (1968) have already pointed out, the magnitudes of the brightest red stars do not appear to depend on the luminosity of their parent galaxy. Observations such as those by Searle and Sargent (1972) suggest that low $\Delta V$ values go with low metal abundance. This view is confirmed by observations of M33. Searle (1971) has shown that the heavy element abundance in this galaxy decreases with increasing distance from its nucleus. This suggests that $\Delta V$ should be smaller in the outer regions of M33 then it is near the nucleus of this galaxy. This is confirmed by Walker (1964) and by Madore (1971) who find that the ratio of the number of bright blue stars to the number of bright red stars decreases with increasing distance from the centre of the Triangulum Nebula. Similarly Hartwick (1970) has been able to show that the ratio of blue to red supergiants in the direction of the galactic centre is greater than it is in the anti-centre direction.

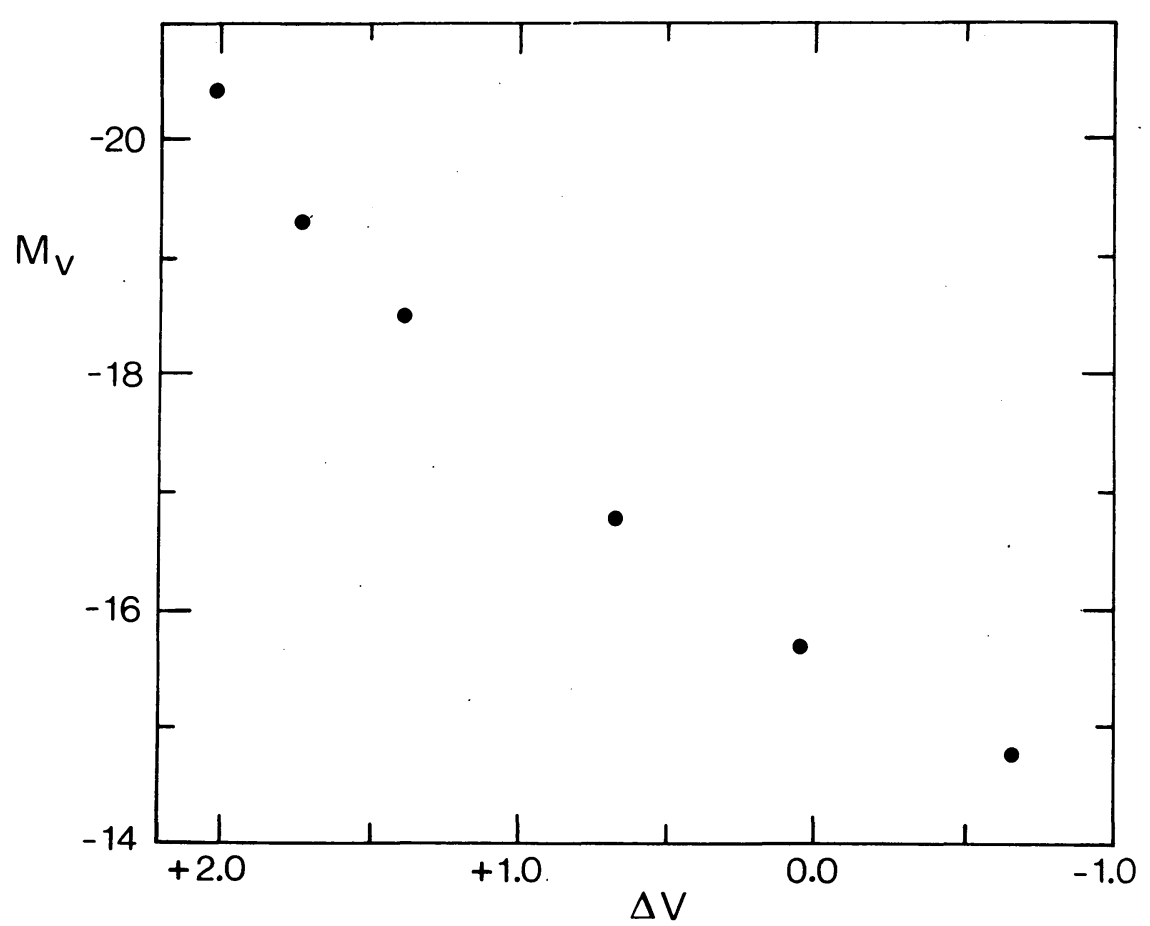

Fig. 2. The figure shows that $\Delta V$, the difference in visual magnitude between the brightest blue and red stars, is strongly dependent on the absolute magnitude $M_{V}$ of the galaxy in which they occur. Since the upper end of the luminosity function of galaxies is very sparsely populated, the very small dispersion in the figure is almost certainly fortuitous. 
In summary it appears that there is now strong observational evidence in support of the conclusions that: (1) chemical composition differences exist between different galaxies and (2) radial composition gradients exist within individual galaxies. The trend of the data is such that galaxies with a high mean density exhibit a higher metal abundance than do galaxies with a low mean density. Similarly, within individual galaxies the regions with the highest heavy element abundance also appear to be the regions of highest density.

\section{Variable Stars and Chemical Abundance}

It has long been known (van den Bergh, 1958) that the mean period of Cepheids interior to the Sun is longer than is that of Cepheids in the anti-centre direction. This observation indicates that Cepheids were formed from interstellar material that had a radial composition gradient. The sense of this gradient suggests that the interstellar gas in the outer part of the galactic disk (where Cepheid periods are similar to those in the Large Magellanic Cloud) has a lower metal abundance than does the gas in the inner region of the Galaxy. Such a trend is strongly supported by the observations of $\mathrm{H}$ II regions in a number of nearby external galaxies. These observations clearly show a higher heavy element abundance in the inner parts of galaxies than they do in their outer regions (Peimbert, 1968; Searle, 1971).

Additional evidence in support of the view that the evolutionary tracks of young high-evolved stars are quite sensitive to differences in chemical composition is provided by the observation that the period-frequency distribution of Cepheid variables in the Magellanic Clouds differs radically from that in the Galaxy. The data in Table IV show that the Cepheids in the Small Magellanic Cloud have a much shorter median period than do those in the Galaxy. The Cepheids in the Large Cloud are seen to have a median period that is intermediate between that of Cepheids in the Galaxy and in the Small Cloud.

TABLE IV

Periods of Cepheids (Gaposchkin, 1971)

$\begin{array}{llll} & \text { Galaxy } & \text { LMC } & \text { SMC } \\ \text { Median } \log P \text { (day) } & 0.78 & 0.64 & 0.42\end{array}$

It is not yet certain whether the period-maximum amplitude relation for Cepheids in the Magellanic Clouds is similar to that for galactic Cepheids. Originally Arp and Kraft (1961) appeared to have shown that the Cepheids in the Magellanic Clouds had a period-maximum amplitude relation different from that which is observed near the Sun. If correct, this conclusion would have far-reaching consequences because it might indicate that the period-luminosity relation of Cepheids is not the same in all galaxies. More recently Shaltenbrand and Tammann (1970) have found that there is no evidence for a systematic difference between the period versus maximum-pulsationamplitude relations in the Galaxy and in the Magellanic Clouds. This question has 
recently been re-examined by Madore (unpublished). His results are plotted in Figures 3 and 4 . These figures show that it is not yet possible to state with certainty whether the period versus pulsation-amplitude relations in the Magellanic Clouds and in the Galaxy are in fact the same.

The Cepheid period-luminosity-amplitude relation of Sandage and Tammann (1971) yields distance moduli $(m-M)_{B}=18.91$ and $(m-M)_{B}=19.35$ for the LMC and SMC respectively. According to Graham (1973), the mean of the median magnitudes of the RR Lyrae variables in these systems are $\langle\dot{B}\rangle=19.22$ in the LMC and $\langle\dot{B}\rangle=19.55$ in the SMC. From these data, $\left\langle M_{\dot{B}}\right\rangle=+0.31$ in the $\mathrm{LMC}$ and $\left\langle M_{\dot{B}}\right\rangle=+0.20$ in the SMC (See Table V). These values are significantly brighter (Sandage, 1972) than the

\section{TABLE V}

Absolute magnitudes of RR Lyrae variables

$\begin{array}{lcr} & \text { LMC } & \text { SMC } \\ (m-M)_{B} \text { (Sandage and Tammann, 1971) } & 18.91 & 19.35 \\ B\rangle(\text { Graham, 1973) } & 19.22 & 19.55 \\ \langle\dot{M}\rangle(\text { RR) } & +0.31 & +0.20 \\ \langle M \dot{B}\rangle \text { (RR) (van Herk, 1965) } & +0.87+0.22\end{array}$

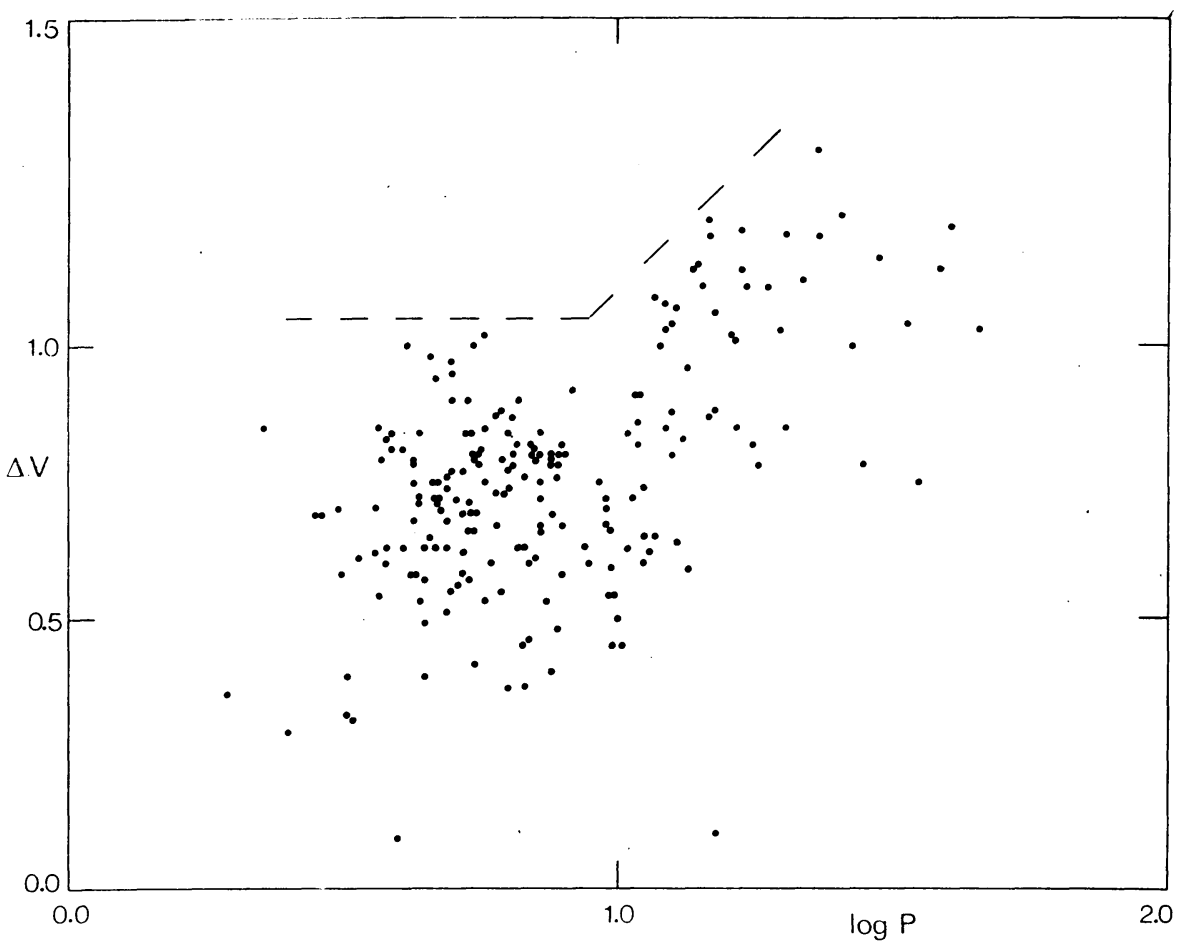

Fig. 3. Period-amplitude relation for galactic Cepheids. The dashed line shows the adopted upper envelope for galactic Cepheids. 


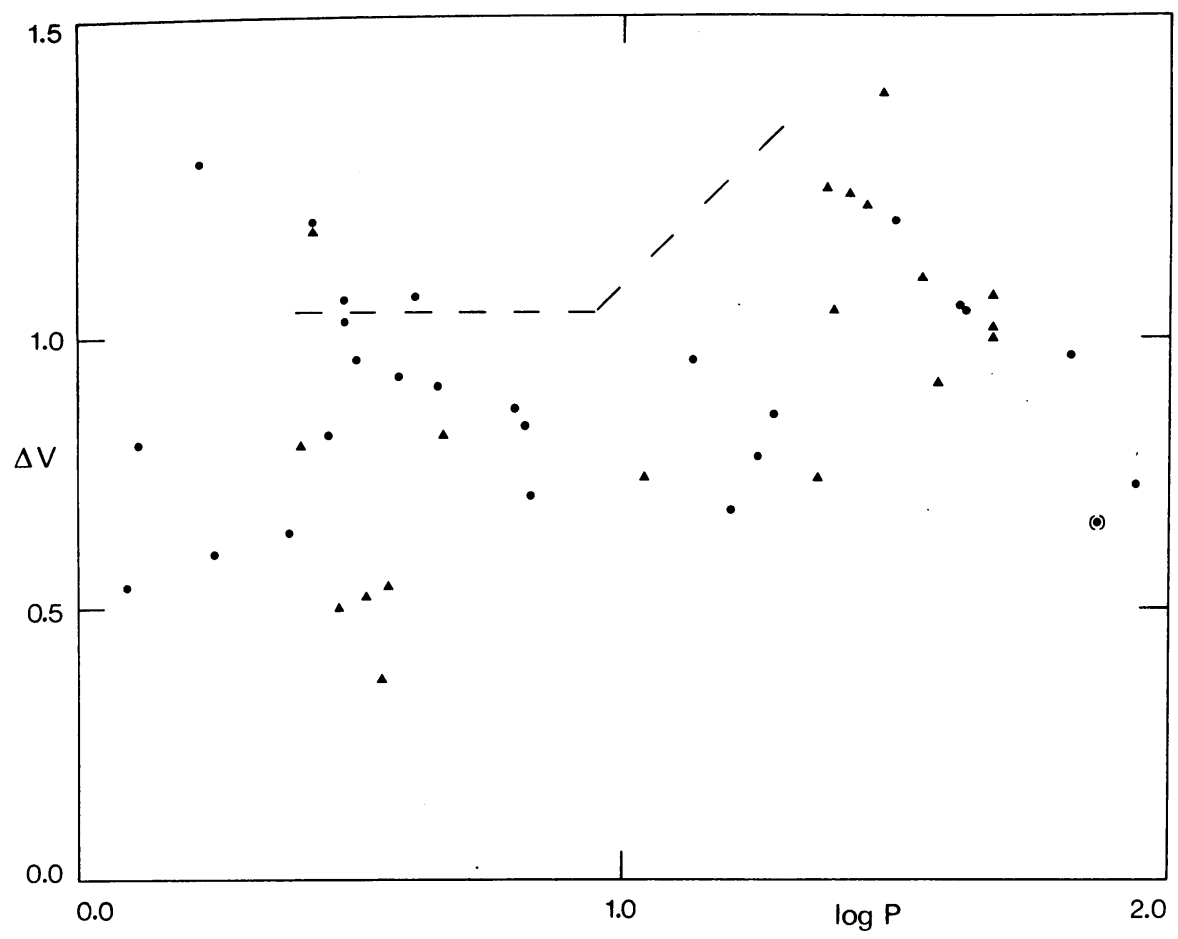

Fig. 4. Period-amplitude relation for the $\operatorname{SMC}(O)$ and $\operatorname{LMC}(\Delta)$. Also shown is the upper envelope to the galactic period-amplitude relation.

value $\left(M_{\dot{B}}\right)=+0.87 \pm 0.22$ m.e. which van Herk (1965) obtained for galactic RR Lyrae variables. Taken at face value, these results indicate that either:

(1) the RR Lyrae variables in the Magellanic Clouds are brighter by $\sim 0.5$ mag than their galactic counterparts or

(2) the Cepheid period-luminosity-amplitude relation in the Clouds is $\sim 0.5 \mathrm{mag}$ fainter than it is in the Galaxy.

I am grateful to Drs Newell and Rood for making unpublished material available to me.

\section{References}

Ables, H. D., Newell, E. B., and O' Neil, E. J.: 1973, Paper Presented at the Los Angeles Meetings of the Astron. Soc. Pacific.

Arp, H. C.: 1958, Astron. J. 63, 487.

Arp, H. C. and Kraft, R. P.: 1961, Astrophys. J. 133, 420.

Castellani, V., Giannone, P., and Renzini, A.: 1973, in J. D. Fernie (ed.), 'Variable Stars in Globular Clusters and in Related Systems', IAU Colloq. 21, 217.

Faber, S. M.: 1973, Astrophys. J. 179, 731.

Gaposchkin, C. P.: 1971, in A. B. Muller (ed.), The Magellanic Clouds, D. Reidel Publ. Co., DordrechtHolland, p. 34.

Gascoigne, S. C. B.: 1962, Monthly Notices Roy. Astron. Soc. 124, 201. 
Gascoigne, S. C. B.: 1966, Monthly Notices Roy. Astron. Soc. 134, 59.

Graham, J. A.: 1973, in J. D. Fernie (ed.), 'Variable Stars in Globular Clusters and in Related Systems', IAU Colloq. 21, 120.

Hartwick, F. D. A.: 1970, Astrophys. Letters 7, 151.

Hodge, P. W.: 1960a, Astrophys. J. 132, 341.

Hodge, P. W.: 1960b, Astrophys. J. 132, 346.

Hodge, P. W.: 1965, Astrophys. J. 142, 1390.

Johnson, H. L. and Morgan, W. W.: 1955, Astrophys. J. 122, 429.

Kayser, S. E.: 1967, Astron. J. 72, 134.

Larson, R. B.: 1969, Monthly Notices Roy. Astron. Soc. 145, 405.

McClure, R. D.: 1969, Astron. J. 74, 50.

McClure, R. D. and van den Bergh, S.: 1968, Astron. J. 73, 1008.

Madore, B. F.: 1971, University of Toronto (M.Sc. Thesis).

Peimbert, M.: 1968, Astrophys. J. 154, 33.

Peimbert, M. and Spinrad, H.: 1970, Astron. Astrophys. 7, 311.

Rood, R. T.: 1973, Astrophys. J. 184, 815.

Sandage, A. R.: 1971, Astrophys. J. 166, 13.

Sandage, A. R.: 1972, Quart. J. Roy. Astron. Soc. 13, 202.

Sandage, A. R. and Tammann, G. A.: 1971, Astrophys. J. 167, 293.

Schaltenbrand, R. and Tammann, G. A.: 1970, Astron. Astrophys. 7, 289.

Searle, L.: 1971, Astrophys. J. 168, 327.

Searle, L. and Sargent, W. L. W.: 1972, Astrophys. J. 173, 25.

Spinrad, H. and Schweizer, F.: 1972, Astrophys. J. 171, 403.

Tammann, G. A. and Sandage, A. R.: 1968, Astrophys. J. 151, 825.

Tifft, W. G.: 1963, Monthly Notices Roy. Astron. Soc. 125, 199.

van Agt, S. L. T. J.: 1967, Bull. Astron. Inst. Neth. 19, 275.

van den Bergh, S.: 1958, Astron. J. 63, 492.

van den Bergh, S.: 1968, J. Roy. Astron. Soc. Can. 62, 145.

van den Bergh, S.: 1969, Astrophys. J. Suppl. 19, 145.

van den Bergh, S.: 1971, Publ. Astron. Soc. Pacific 83, 663.

van den Bergh, S.: 1972, Astron. Astrophys. 20, 469.

van Herk, G.: 1965, Bull. Astron. Inst. Neth. 18, 71.

Walker, M. F.: 1964, Astron. J. 69, 744.

Walker, M. F.: 1970, Astrophys. J. 161, 835.

Walker, M. F.: 1972a, Monthly Notices Roy. Astron. Soc. 156, 459.

Walker, M. F.: 1972b, Monthly Notices Roy. Astron. Soc. 159, 379.

\section{DISCUSSION}

Graham: The present data do not indicate any difference between the mean absolute magnitude of the Magellanic Cloud RR Lyrae stars and that of the RR Lyraes in the Galaxy. I find that a time averaged mean visual magnitude of $+0 m 5$ seems to fit well in all three cases (IAU Colloq. 21, 1973).

van den Bergh: The data in Table $\mathrm{V}$ give the median magnitudes that you obtained for the RR Lyrae variables in the globular clusters in the Magellanic Clouds. As you point out, these values are probably too bright by 0.1 or $\mathbf{0 . 2}$ mag due to the effects of background enhancement. This small overestimate of the luminosity of the RR Lyrae variables in the Clouds is almost exactly compensated for by the fact that photoelectric observations of galactic RR Lyrae variables (Fitch, W. S., Wisniewski, W. Z., and Johnson, H. L.: 1966, Lunar and Planetary Lab. Communications No. 71) show that van Herk (1965) used a magnitude system which was about $0.2 \mathrm{mag}$ too bright for the galactic RR Lyrae stars. There remains therefore a discrepancy of between two and three standard deviations between the absolute magnitudes of the galactic RR Lyrae stars and the absolute magnitudes of the cluster type variables in each of the Clouds that is obtained by using the period-luminosity-amplitude relation of Sandage and Tammann (Astrophys. J. 167, 293, 1971). This discrepancy is virtually removed if the period-luminosity-colour relation (Sandage, A. R. and Tammann, G. A.: 1969, Astrophys. J. 151, 531), rather than the period-luminosity-amplitude relation, is used to estimate the distance moduli of the Clouds. According to Sandage and Tammann (1969) the P-L-C relation yields distance moduli 
$(m-M)_{B}$ of 18.65 and 19.05 for the $\mathrm{LMC}$ and SMC respectively. These values yield $\left\langle M_{\dot{B}}\right\rangle_{\mathrm{RR}}=0.57$ for the LMC and $\left\langle M_{B}\right\rangle_{\mathbf{R R}}=0.50$ for the SMC, values which differ only marginally from van Herk's luminosity for galactic RR Lyrae variables. The most straightforward interpretation of this result is that the period-luminosity-colour relation for cepheids yields a more nearly correct distance to the Clouds than does the period-luminosity-amplitude relation.

Gascoigne: It has been suggested by Christy (1971, in A. Muller (ed.), The Magellanic Clouds, D. Reidel Publ. Co., p. 136) and Iben (Ann. Rev. Astron. Astrophys. 5, 606, 1967) that the SMC cepheids are metal-deficient relative to those in the Galaxy. Changing the metal content changes the mass-luminosity and $T_{\text {eff }}-(B-V)$ relations, and it can be shown that changing $Z$ from 0.02 to 0.005 makes cepheids at a given period and colour fainter by $0.3-0.4 \mathrm{mag}$. In the present context this brings the SMC nearer by about $0.35 \mathrm{mag}$ in the modulus.

The observed PLC relations for cepheids in the Galaxy or Magellanic Clouds are satisfied with a scatter better than $0.1 \mathrm{mag}$, observational errors included. This implies a very high degree of chemical homogeneity in these cepheids.

Sargent: There are two pieces of evidence regarding the possible existence of young galaxies which you did not discuss. The first concerns Ring Galaxies, of which about one dozen are known. These are galactic sized objects with diameters of around $10 \mathrm{kpc}$. They have no optical nucleus; the rings appear to be composed of giant $\mathrm{H}$ II regions. Kinematic work (unpublished) by Lynds, by Speigel and Thys and by myself shows that the rings are expanding (or contracting, depending on which side is closest) on a timescale of about $10^{8} \mathrm{yr}$. Recent studies of two rings by Searle and myself (unpublished) also indicate a time scale of a few time $10^{8} \mathrm{yr}$ for the dominant stellar populations of these objects.

The second piece of evidence concerns compact clusters of galaxies - Seyfert's Sextet is a typical example - in which the galaxies are almost touching one another. In such systems the timescale for the galaxies to collide and, presumably, to tear one another apart, is much less than the Hubble time. Aspects of this problem have been discussed by Peebles in his book Physical Cosmology.

van den Bergh: Cannot Seyfert's Sextet be interpreted as a cluster, initially larger, which has ejected some of its members and thereby lost kinetic energy, so shrinking to its present size?

Sargent: Numerical experiments suggest that the probability of this being the case is very low.

Tifft: Do not Seyfert's Sextet galaxies show spectral characteristics of old stars - i.e. strong HK absorption lines?

Sargent: Yes, some of the galaxies show emission lines in addition to $\mathrm{H}$ and $\mathrm{K}$ absorption from old stars, but generally the spectra give no sign that the galaxies are young.

Heidmann: We made 21-cm line observations of twelve Haro galaxies (Astron. Astrophys. 29, 217) and found a very strong correlation between mean optical surface brightness and neutral hydrogen mass-to-luminosity ratio: when the brightness varies from 19 to $21 \mathrm{mag}(\operatorname{arc~sec})^{-2}$, this ratio increases from 0.03 to 1.0 in solar units. We think this could be accounted for by flashes of stars occurring in Haro galaxies, such as those advocated by Sargent and Searle for Zwicky compact galaxies.

Lewis: What effect does your work on the absolute magnitudes of bright blue stars and the periodluminosity relation of cepheids have upon their role as distance indicators to nearby galaxies such as NGC 2403?

van den Bergh: Since there is now some suspicion that the cepheid period-luminosity relation may depend on chemical composition, it would be most prudent to compare the galactic period-luminosity relation only with that in other luminous galaxies of types $\mathrm{Sb}$ or Sc.

Present evidence seems to suggest that bright blue variables may form a continuum between the brightest stable main sequence stars and supernovae of type II. If this conclusion is correct then the blue Hubble-Sandage variables are not suitable as precision distance indicators. 